\title{
Do leaders have to be lions?
}

\author{
$\operatorname{liaoyu}^{1, \mathrm{a}, *}$ \\ ${ }^{1}$ City College of Wuhan University of Science and Technology, Wuhan 430083, China \\ atomliao-898@163.com \\ *corresponding author
}

Keywords: The lion, leader, Competency, Strategy

\begin{abstract}
With the development of China's social and economic progress, In recent years, the demand for leadership from all walks of life develops rapidly. The most influential in the selection of government leaders is the so-called "lion" leadership, But good leaders are never a single type. This article analysis the quality of the leader, discussed the strategy of selecting the right leader.
\end{abstract}

\section{Introduction}

Leadership is a kind of behavior or behavior process that leaders exert influence on their subordinates in order to realize the goal of organization ${ }^{[1]}$.

A leader is the individual and collective who holds a certain leadership position in a formal social organization, performs a certain leadership function, has a certain power, and has some kind of leadership responsibility. There is a saying in the field of management: A pack of sheep led by a wolf can defeat a pack of wolves led by a sheep. This statement shows the importance of a leader and implies the power of a team. Leaders should properly use the power factor and the non - power factor, establish authority to make the members of the organization cohere. Leaders should be strict with themselves and lead by example to lead, influence and promote the members to improve their work, and strive to achieve their common goals.

\section{What is the lion leader?}

According to Netease news report in May 18, 2017: Chen Yixin who is the Secretary of Wuhan Municipal Committee, chaired the congress of the municipal Standing Committee, deliberated and passed the large research program of selecting the " lion type" cadres. There will find and master 100 " lion - shaped" cadres in the city, and select them to the top - level positions before the end of this month. To this end, 17 research groups in Wuhan city launched a large research to 137 units ${ }^{[2]}$.

According to the Yangtze daily news, the " portrait " of " lion type" cadre is named by Chen Yixin: the outstanding cadres is Loyal and clean, ideological liberation, open-minded, dare to fight, dare to bear the responsibility.

The " lion type" cadre was first proposed by Zhejiang province, In 2013, Xia baolong who is the party Committee secretary in Zhejiang province proposed to create a " lion model" cadre teambuilding. In the next few years," the cadres of the lion type" are allocated to the top leaders, which has become an important guidance for the cadres construction in Zhejiang province.

\section{The competency of a good leader}

Quality refers to the general name of the relatively stable psychological quality and accomplishment, cultivation and ability formed by the internalization of knowledge, on the basis of human being's congenital physiology, through the influence of the acquired education and social environment. Quality is the starting point of judging a person's ability to work, is the individual characteristic that decides and distinguishes performance difference. 


\subsection{Quality iceberg model theory}

David C. McClelland, a famous American psychologist, presented a famous quality iceberg model in 1973, The so-called " iceberg model" is to divide the different expression tables of individual quality into " above of the iceberg " and " below of the iceberg "

Among them, the " above of the iceberg " includes basic knowledge, basic skills, and external performance, which is easy to understand and measured, and relatively easy to change and develop through training.

The " below of the iceberg " include social roles, self-image, characteristics and motivation, which are intrinsic and difficult to measure. They are not very easy to change by outside influence, but they play a key role in the behavior and performance of people.

Table 1 Quality hierarchy structure

\begin{tabular}{|c|c|}
\hline Quality level & Definition \\
\hline Skills & The ability of a person to complete a task or task. \\
\hline Knowledge & A person's understanding of a particular field. \\
\hline Role orientation & It refers to what a person expects of a career, what a person wants to do. \\
\hline Values & The value orientation of a person to the right and wrong, importance, necessity, etc. \\
\hline Self - cognition & A person's awareness and opinion of himself. \\
\hline Quality & A continuous and stable behavior of a person. \\
\hline Motivation & $\begin{array}{c}\text { Refers to the natural and continuous thoughts and preferences of one person, driving, } \\
\text { guiding, and deciding individual action. }\end{array}$ \\
\hline
\end{tabular}

Iceberg model provides a new perspective and a more advantageous tool for the practice of human resource management, it not only meets the requirements of modern human resource management, constructs the competency model of certain post, makes clear description for the competency of a job, and becomes an important basis for personnel quality assessment, and provides the scientific premise for the development of human resource management.

\subsection{Quality structure of leadership talent}

According to the iceberg model theory, we need to follow a certain steps to analyze the quality structure of leadership talent.

First of all, different types of work, quality requirements are different, to determine which quality is the required quality of the type of work.

Secondly, after determining the competency, the organization should establish an evaluation system that can measure the level of individual competency, this evaluation system should also be tested by objective data, and can distinguish the work performance.

Finally, on the basis of accurate measurement, the results and application of competency assessment is designed.

The strength of leadership depends on the quality and ability of the leader. The auxiliary conditions are the environment and subordinate factors. The quality is a certain persistence character and moral character owned by the leader, can get the social identity; Ability is the behavior of the leader through the combination of his own wisdom and practical experience. Quality depends on the leader's values, it has a congenital genetic factor, but more is acquired from the nurture, such as family education, school education, social customs influence. Character, character, and health are the basic elements of quality. The ability depends on the leader's intelligence, knowledge, experience and strain level. It is closely related to the quality, which is the comprehensive reflection of learning ability, communication ability, insight ability and motivation ability. The combination of different qualities and abilities determines the strength of leadership.

According to the iceberg model theory, the quality structure of a good leader can be broken down into the following: 


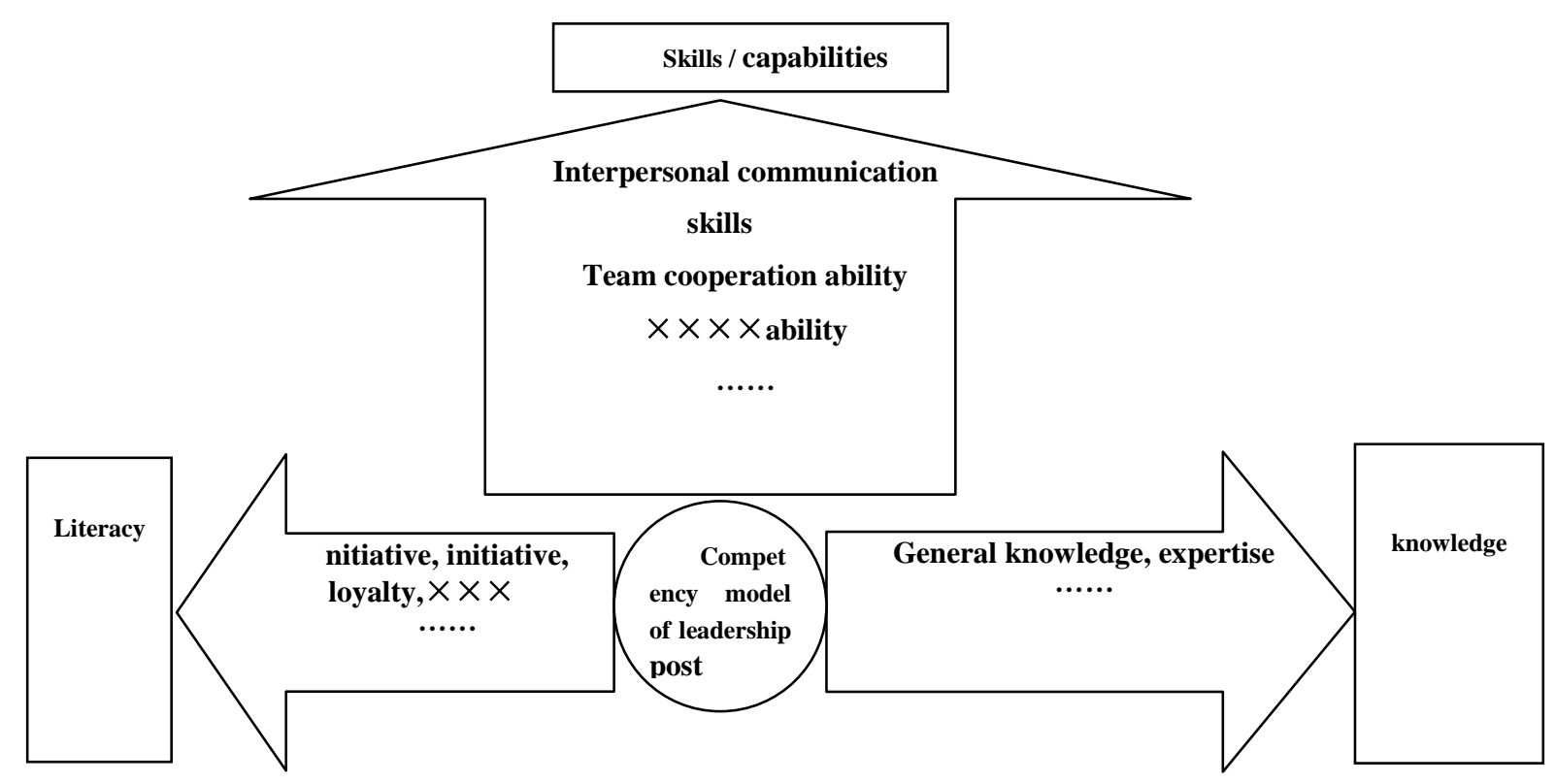

Figure 1 Competency model of leadership post

\section{The strategy of scientific selection of appropriate leadership talents}

So how to select leaders scientifically? I think that there are the following steps:

\subsection{Constructing competency model of excellent leaders}

(1) Clear organizational development strategic objectives

The development strategy objective of the organization is to establish the overall guiding principle of job competency model, human resource managers should first analyze the key factors affecting the realization of organizational strategy, research the competition and challenges, and then extract the competency of organization leaders, so as to build up competency model conforming to the organizational culture and environment.

(2) Identify target posts

The implementation of organizational strategic planning is closely related to the key positions in the organization. therefore, the core leadership positions that play a key role in the organizational strategic objectives should be selected as the target position in the establishment of competency model, and then the competency characteristics required by the leaders should be analyzed, so as to build up competency model conforming to the post characteristics.

(3) Define the merit standard of target post

The perfect performance appraisal system of the organization is to define the basis of merit standard. Through comprehensive evaluation of the elements of the target leadership position, distinguish the performance of leaders in the target post performance, ordinary performance or poor performance, in order to define the standard of merit, and then define good performance standard decomposition into specific tasks, so as to identify the behavior characteristics of leaders to produce outstanding performance.

(4) Select sample group

According to the competency of target post, the performance excellent staff ( $3 \sim 6$ ) and the general performance leader ( $2 \sim 4$ ) are selected randomly among the leaders who are engaged in the job.

(5) Collect and sort data information

The collection and collation of data information is the core work of constructing competency model, generally through the behavioral event interview, expert database, questionnaire survey 
method to obtain competent characteristics data in the sample group, The information obtained will then be collated and classified.

(6) Define the competency of the post

According to the target post data, we focus on the behavior process or fragments which have significant influence on the key behavior, characteristics, thoughts and feelings of leaders in the actual work, and identify the differences between the leaders and the general performance leaders in handling the similar events, identify the ability quality of the key behaviors and the results, and define the competency standards.

(7)Divide up competency level

After all the items of target post competency are defined, the quality items should be classified according to each quality project, and the behavior description will be made to different quality levels, and competency model will be established preliminarily.

(8)Constructing competency model

Combining the actual situation of organizational development strategy, environment and target position, the established competency model is matched and balanced in organization, position and leader, and the competency model is constructed and continuously improved.

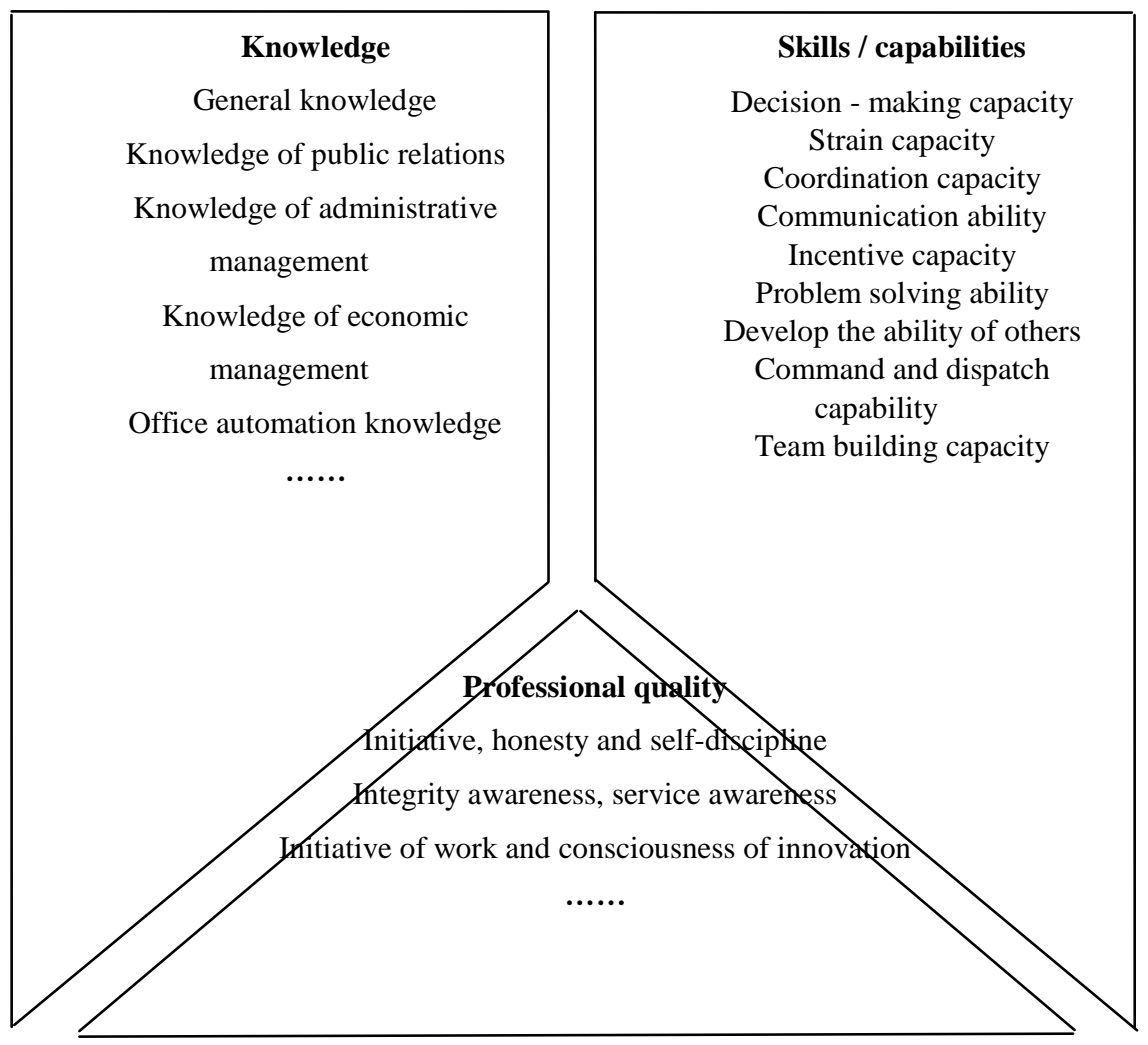

Figure 2 On the segmentation index system of leadership competency model

\subsection{Establishment of the selection evaluation team}

The evaluation team is composed of organizational human resources leadership, recruitment unit leadership, senior leadership and evaluation experts, to determine the division of labor and work schedule. The evaluation expert listens to the introduction of the organization leadership and the relevant personnel, the evaluation team carries out the discussion, consults with the information of the leading position and analyzes the job responsibility and norm, prepares for the talent selection with the competency model.

\subsection{Selecting evaluation method and developing evaluation tools}

For example, according to the requirements of an organization to a good leader, we can build a competency model consisting of 10 competency quality in the posts and assign weights to different 
qualities depending on the importance of the work.

Table 2. 10 competency weight list for leaders

\begin{tabular}{|c|c|c|}
\hline Quality element of competency & $\begin{array}{c}\text { Important degree of investigation } \\
\text { score }\end{array}$ & weight \\
\hline Knowledge & 8 & $8 / 66.5=12.03 \%$ \\
\hline Skills & 7 & $7 / 66.5=10.53 \%$ \\
\hline $\begin{array}{c}\text { Organizational planning capacity } \\
\text { Persuasive communication skills }\end{array}$ & 8 & $8 / 66.5=12.03 \%$ \\
\hline $\begin{array}{c}\text { The ability of building interpersonal } \\
\text { relation }\end{array}$ & 7.5 & $\begin{array}{c}7.5 / 66.5=11.28 \\
\%\end{array}$ \\
\hline $\begin{array}{c}\text { Team building and collaboration } \\
\text { capability }\end{array}$ & 7 & $7 / 66.5=11.28 \%$ \\
\hline Ability of thinking analysis & 6.5 & $6.5 / 66.5=9.77 \%$ \\
\hline Decisive decision - making capacity & 6 & $6 / 66.5=9.02 \%$ \\
\hline Service consciousness & 5.8 & $5.8 / 66.5=8.72 \%$ \\
\hline onsciousness of innovation & 5.5 & $5.5 / 66.5=8.27 \%$ \\
\hline Total score & 5.2 & $5.2 / 66.5=7.82 \%$ \\
\hline
\end{tabular}

According to the characteristics of the above 10 evaluation factors, the evaluation team decided to select four candidates from eight candidates to the second round with professional knowledge test and psychological test, and then organized four people to carry out leadership group discussion, role play, official document test, and finally decided the winner. If you encounter two candidates who are evenly divided, you can use structured interviews to select the candidates. At the same time, combined with the results of its original work results to select.

\subsection{Implementation of selection and evaluation}

(1) Operational specification of training evaluation team members

The training content includes the standard implementation assessment protocol (the main test should be done and unfavorable things), standard guide language ( the main test to be said ), and the time control in the process, on-site emergency treatment, etc.

(2) Preparation of sites, equipment and materials required

The evaluation site requires good lighting and no noise, and the desks and chairs can be arranged reasonably in space.

The testing equipment includes whiteboard, audio and video projection equipment and camera device, etc.

The materials required for the measured personnel include the test number, the paper, the answering paper, scratch paper paper, pencil and eraser, etc.; The materials required for the testing personnel include evaluation manual, recording paper and timer, etc.

(3) Specific implementation programme

The specific process of implementation is shown in the following figure. 


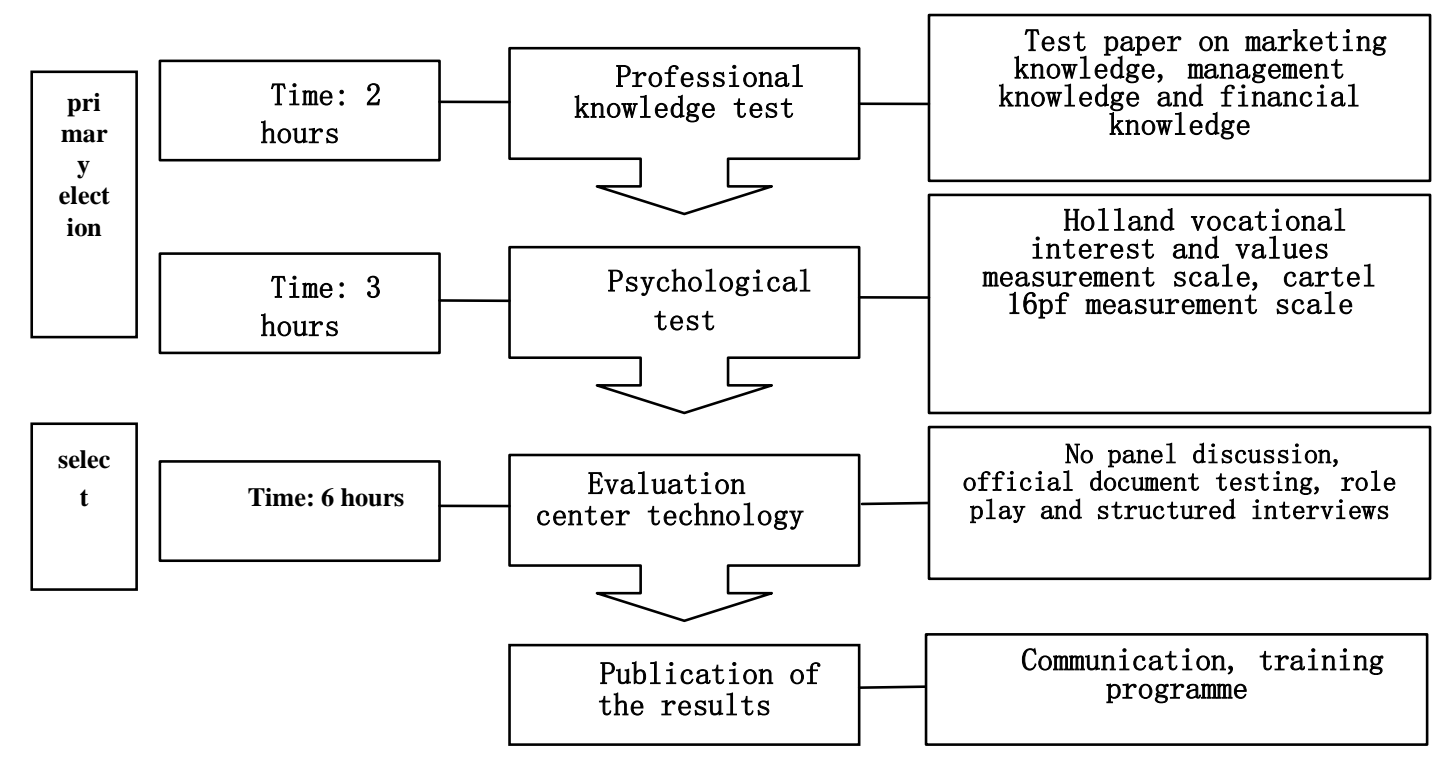

Figure 3 Schematic diagram of evaluation implementation scheme

\section{Conclusions}

To sum up, through this scientific selection and evaluation mode, we can truly promote the outstanding leaders who meet the working position conditions to the right positions and provide better leadership service for the development of our economic society.

\section{References}

[1] 360baike. http://baike.so.com/doc/5296612-5531266.html.

[2] Netease news. http://news.163.com/17/0521/09/CKUUUTN00001875N.html.

[3] Wangshuhong. personnel quality evaluation, Beijing university press,2012.7 